\title{
IN-PLANE FREE VIBRATION OF TWO-MEMBER OPEN FRAME WITH TRANSVERSE CRACK WITH END MASS
}

\section{MAMATHA Y P, BHANUPRAKASH M J, PRAVEENKUMARA B M \& RAJESH KUMBARA S K}

Department of Mechanical Engineering, VidyavardhakaOCollegeOof Engineering, Mysuru, India

\begin{abstract}
This paper focus on addressing the problem of0In-plane free vibration analysis0of two-member open0frame with transverse0crack. A two-member0open frame is0modelled using0Euler-Bernoulli beam0theory. The angle between the beams is $90^{\circ}$ and payload is attached at the end0of the second0beam. TheOlinearized equation of0motion is derived using0Hamilton's principle0and the0general frequency equations are presented with crack with two member open frame. Transverse vibration0of a frame in the0presence of a normal edge0crack is considered. The crack is modelled using the concept of rotational spring. The determination0of natural frequencies0for a given crack0location and0size is a straightforward problem and is the aim0of project work. Numerical results are tabulated for both the cases (i,e without and with end mass) and graphs are drawn.

KEYWORDS: Dynamic Behavior, Crack, Modal Parameter, Vibration
\end{abstract}

Received: Jun 08, 2020; Accepted: Jun 28, 2020; Published: Sep 19, 2020; Paper Id.: IJMPERDJUN20201339

\section{INTRODUCTION}

In a structural member crack introduces local flexibility due to strain energy concentration under load at the vicinity of the crack tip. This depends on crack depth . the dynamic behavior of the structure changes due to flexibility and from this the position of crack and magnitude can be identified. It is known that vibrational parameters changes when there is a crack in the structure e.g. reduction in stiffness, increase in the damping etc, and these parameters are mode dependent .They are natural frequencies, modal damping values and mode shapes etc.

\section{LITERATURE SURVEY}

Using Hu-Washizu-Barr variational formulation the differential equation and the boundary conditions of the cracked beams and compared the local flexibility method. The crack location and depth was measured using vibration amplitude by Rizos et al [2]. A Dado [3] developed an algorithm to predict the depth and location of cranked shaft. Here Frobenious technique is used to model the crack.

Jialou $\mathrm{Hu}$ and Robert [4] have used massless springs to represent cracks and the continuum damage concept is used for crack detection.

B P. Nandwana and S. K. Maiti[5] have used measument of natural frequencies for the detection of crack size and location in a stepped cantilever beam.

B P. Nandwana and S. K. Maiti[6] have used rotational spring concept for modeling inclined and internal crack in a slender beam. 


\section{DESCRIPTION OF THE SYSTEM}

The system is mainly composed of 2 flexible beam members and a payload which are arranged as shown in figure (1). The first beam is vertical, while the second beam is attached to the tip of the first beam such that it is inclined at an angle of $90^{0}$ from the vertical. The payload is attached at the end of the second beam and the motion of the system is assumed to be a planar and the case (1) crack is located in vertical segment and case (2) crack is located in vertical segment and case (2) crack is located in horizontal segment.

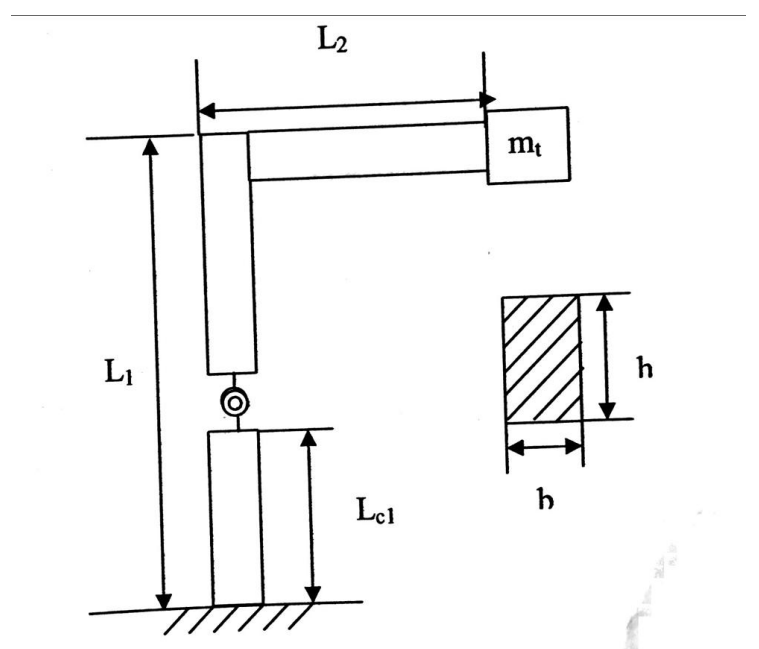

Figure 1: Two Frame Open Frame(L-frame) Structure with End Masses and Crack is in Verticle Segment.

\section{Case 1: Crack Located in Vertical Segment}

Using Euler Bernoulli theory the equations of motion can be written as $\lambda \lambda$

$$
E I \partial^{4} v_{i} / \partial x_{i}^{4}+p L i \partial^{2} v_{i} / \partial t^{2}=0
$$

In which E is Young's Modulus of elasticity, $\mathrm{I}$ is the second moment of area of cross- section $\mathrm{p}$ is the density of material, $v_{i}$ is the transverse displacement and $x_{i}$ is the axial coordinate for the segment $i$. In the above the torsional oscillation of a segment has been ignored.

The general solution of equation (1) can be written in the form.

$$
v_{i\left(x_{2}, r\right)=L_{i}} v_{i}\left(\epsilon_{i}\right) i \omega t
$$

In which $\omega$ is the natural frequency, $\boldsymbol{\epsilon}_{i}=\mathrm{x}_{\mathrm{i}} / \mathrm{L}_{\mathrm{i}}$ and $\mathrm{L}_{\mathrm{i}}$ is the length of the segment $\mathrm{i}$.

Using equation (1) and (2)

The equations of motion can be written as

$$
\begin{aligned}
& \partial^{4} V_{1} / \partial \epsilon_{1}^{4}-\lambda_{1}^{4} V_{1}=0 \ldots \ldots \ldots \ldots \ldots .0 \leq \in^{1} \leq \beta_{1} \\
& \partial^{4} V_{2} / \partial \epsilon_{1}^{4}-\lambda_{1}^{4} V_{2}=0 \ldots \ldots \ldots \ldots \ldots \beta_{1} \leq \epsilon_{1} \leq 1 \\
& \partial^{4} V_{3} / \partial \epsilon_{2}^{4}-\lambda_{2}^{4} V_{3}-\epsilon_{2} V_{2}(1)=0 \ldots \ldots \ldots \ldots \ldots .0 \leq \epsilon_{1} \leq 1
\end{aligned}
$$


Where, $\beta_{1}=L_{C 1} / L_{1}, \varepsilon_{1}=x / L_{1}, \varepsilon_{2}=x / L_{2}$

$$
\lambda_{1}=\rho L_{1} 4 \omega^{2} / E I_{s}, \lambda_{2}=\rho L_{12} 4 \omega^{2} / E I_{s}
$$

The general solution to the equation of motion is given by

$$
\begin{aligned}
& V_{1}\left(\varepsilon_{1}\right)=A_{1} \sin \lambda_{1} \varepsilon_{1}+B_{1} \cos \lambda_{1} \varepsilon_{1}+C_{1} \sin \lambda_{1} \varepsilon_{1}+D_{1} \cos \lambda_{1} \varepsilon_{1} \\
& V_{2}\left(\varepsilon_{1}\right)=A_{2} \sin \lambda_{1} \varepsilon_{1}+B_{2} \cos \lambda_{1} \varepsilon_{1}+C_{2} \sin \lambda_{1} \varepsilon_{1}+D_{2} \cos \lambda_{1} \varepsilon_{1} \\
& V_{3}\left(\varepsilon_{2}\right)=A_{3} \sin \lambda_{2} \varepsilon_{2}+B_{3} \cos \lambda_{2} \varepsilon_{2}+C_{3} \sin \lambda_{2} \varepsilon_{2}+D_{3} \cos \lambda_{2} \varepsilon_{2}-v_{2}-(1)
\end{aligned}
$$

Where $A_{i}, B_{i}, C_{i}$ and $D_{i}$ are arbitrary constants, which are determined from the boundary conditions.

The boundary conditions are at the fixed and free end, continuity of displacement, moment, shear forces at the crack location, jump condition in the slope and compatibility conditions can be written as follows.

The boundary conditions at the fixed end slope are zero.

$\mathrm{V}_{1}(0)=0$

$\mathrm{V}_{1}(0)=0$

The compatibility conditions of the displacement, slope, moment and shear forces at the crack location are

$$
\text { At } \varepsilon_{1}=\beta_{t}
$$

The continuity of the deflection at the crack is given by

$$
V_{1}\left(\beta_{1}\right)-V_{2}\left(\beta_{1}\right)=0
$$

At the crack location, the slope of deflection is discontinuous; the values of the slope at both sides of the crack are related to the crack stiffness by.

$$
V_{1 t}\left(\beta_{1}\right)+\left(E_{1} L_{1} / K_{t} L_{1}\right) V_{1}\left(\beta_{1}\right)-V_{2}\left(\beta_{1}\right)=0
$$

From the theory of linear elastic fracture mechanics, the rotational spring stiffness $K_{t}$ is given by $K_{t}=E_{1} b h^{2} / 72 \pi(\alpha / h)^{2} f(a / h)$

Where,

$\mathrm{f}(\mathrm{a} / \mathrm{h})=0.6384-1.035(\mathrm{a} / \mathrm{h})+3.7201(\mathrm{a} / \mathrm{h}) 2-5.1773(\mathrm{a} / \mathrm{h}) 4-7.3324(\mathrm{a} / \mathrm{h})^{5}+2.4909(\mathrm{a} / \mathrm{h})^{6}$

Where,

$\mathrm{K}=\left(\mathrm{K}_{\mathrm{t}} \mathrm{L}_{1} / \mathrm{E}_{1} \mathrm{I}_{1}\right)$

Is the non-dimensional stiffness of the rotational spring representing the crack.

The internal moment at the crack location is same for the two segments and that leads to

$V_{1}\left(\beta_{1}\right)-V_{2}\left(\beta_{1}\right)=0$ 
The shear compatibility equation at the crack location is given by

$$
V_{1}\left(\beta_{1}\right)-V_{2}\left(\beta_{1}\right)=0
$$

The boundary conditions at

At $\left(\varepsilon_{1}\right)=1$ and $\left(\varepsilon_{2}\right)=0$ with end mass is given by

$$
\begin{aligned}
& \mathrm{V}_{3}(0)=0 \\
& \mathrm{~V}_{3}^{1}(0)=0 \\
& \lambda_{1}^{4} \rho L \int_{0}^{1} \varepsilon 2\left\{V_{3}+\varepsilon_{2} V_{2}(1)\right\} d \varepsilon_{2}+\lambda_{1}^{4} M_{t} L^{2}\left\{V_{2}(1)+\left(V_{3}(1)\right\}-V_{2}^{\prime \prime}(1)=0\left(V_{3}(1)\right\}-V_{2}{ }^{\prime \prime}(1)\right. \\
& =0
\end{aligned}
$$

$$
\lambda_{1}^{4} \rho L^{2} \int_{0}^{1}\left\{1 / L V_{2}(1)\right) d \varepsilon_{2}+\lambda_{1}^{4} M_{\varepsilon}\left\{1 / L V_{2}(1)\right\}+V_{2}^{\prime \prime}(1)=0
$$

The boundary conditions at

$\mathrm{At}=\varepsilon 2=1$ with end mass is given by

$\mathrm{V}_{3}{ }^{11}(1)=0$

$$
\lambda_{1}^{4} M_{t}\left\{V_{3}(1)+V_{2}(1)\right\}+\rho L\left\{V_{3}(1)=0\right.
$$

Using the above boundary and compatibility conditions twelve homogeneous equations involving the unknown arbitrary constants are obtained. These can be expressed conveniently in the following form.

$[\mathrm{A}]_{12 * 12}\{\mathrm{q}\}_{12 * 1}=\{0\}_{12 * 1}$

Where $\{q\}=\left[A_{1}, B_{1}, C_{1}, D_{1}, A_{2}, B_{2}, C_{2}, D_{2}, A_{3}, B_{3}, C_{3}, D_{3}\right]^{T}$

The non-zero elements of the matrix $[\mathrm{A}]$ are listed below. The terms contain the natural frequency parameters $\lambda$. For a non-trivial solution for $\{\mathrm{q}\}$

$\operatorname{Det}[\mathrm{A}]=0$

This gives the characteristic equation. 


\section{Case: 2: Crack Located in Horizontal Segment}

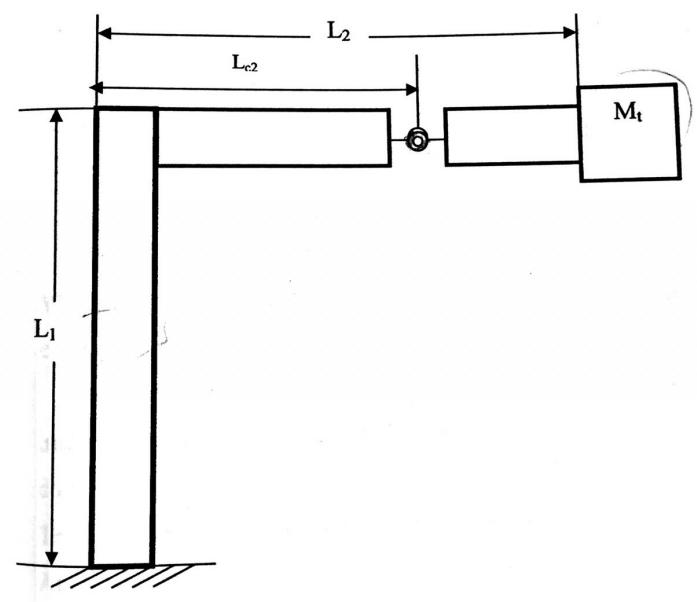

Figure 2: Two Frame Open Frame (L-Frame) Structure with End Masses and Crack is in Horizontal Segment.

Fig. 2 Two Frame Open Frame (1-Frame) structure with end mass and crack is in horizontal segment

Here it is assumed the crack is being located in horizontal segment.

Using Euler - Bernoulli beam theory the equations of motion can be written as

$\mathrm{E}_{\mathrm{i}} \mathrm{I}_{\mathrm{i}} \partial^{4} \mathrm{v}_{\mathrm{i}} / \partial \mathrm{x}_{\mathrm{i}}^{4}+\rho_{\mathrm{i}} \mathrm{L}_{\mathrm{i}} \partial^{2} \mathrm{v}_{\mathrm{i}} / \partial \mathrm{t}^{2}=0$

In which E is Young's Modulus of Elasticity, I is the second moment of area of cross section, $\rho$ is the density of material, $\mathrm{v}_{\mathrm{i}}$ is the transverse displacement and $\mathrm{x}_{\mathrm{i}}$ is the axial co-ordinate for the segment $\mathrm{i}$. In the above the torsional oscillation of a segment has been ignored.

The general solution of equation (18) can be written in the form

$\mathrm{V}_{\mathrm{i}}\left(\mathrm{x}_{\mathrm{i}, \mathrm{t}}\right)=\mathrm{L}_{\mathrm{i}} \mathrm{V}_{\mathrm{i}}\left(\varepsilon_{\mathrm{i}}\right)^{\mathrm{j} \omega \mathrm{t}}$

In which $\omega$ is the natural frequency, $\varepsilon i=x i / L_{i}$ and $L_{i}$ is the length of the segment $i$

Using equations (18) and (19)

The equations of motion can be written as

$$
\begin{array}{ll}
\partial^{4} V_{1} / \partial €_{1}{ }^{4} \cdot-\lambda_{1}{ }^{4} V_{1}=0 & \text {--------- } 0 \leq €_{1} \leq 1 \\
\partial^{4} V_{2} / \partial €_{2}{ }^{4} \cdot-\lambda_{1}{ }^{4} V_{2}-€_{2} V_{i}(1)=0 & \text {-------- } 0 \leq €_{2} \leq \beta_{2} \\
\partial^{4} V_{3} / \partial €_{2}{ }^{4} \cdot-\lambda_{2}{ }^{4} V_{3}-€_{2} V_{i}(1)=0 & \text {-------- } \beta_{2} \leq €_{2} \leq 1
\end{array}
$$

Where $\beta_{2}=\frac{L_{c 2}}{L_{2}}, \xi_{1}=\frac{x}{L_{1}} \xi_{2}=\frac{x}{L_{2}}$

$$
\lambda_{1}=\rho \mathrm{L}_{1}^{4} \frac{\omega^{2}}{\mathrm{El}}, \lambda_{2}=\rho \mathrm{L}_{2}^{4} \frac{\omega^{2}}{\mathrm{El}}
$$

The general solution to the equation of motion is given by 


$$
\begin{aligned}
& \mathrm{V}_{1}\left(\xi_{1}\right)=\mathrm{A}_{1} \sin \lambda_{1} \xi_{1}+\mathrm{B}_{1} \cos \lambda_{1} \xi_{1+} \mathrm{C}_{1} \sinh \lambda_{1} \xi_{1}+\mathrm{D}_{1} \cosh \lambda_{1} \xi_{1} \\
& \mathrm{~V}_{2}\left(\xi_{2}\right)=\mathrm{A}_{2} \sin \lambda_{2} \xi_{2}+\mathrm{B}_{2} \cos \lambda_{2} \xi_{2}+\mathrm{C}_{2} \sinh \lambda_{2} \xi_{2}+\mathrm{D}_{2} \cosh \lambda_{2} \xi_{2}-\xi_{2} \mathrm{~V}_{1}(1) \quad \text { (21b) } \quad \mathrm{V}_{3}\left(\xi_{2}\right)=\mathrm{A}_{3}
\end{aligned}
$$

$\sin \lambda_{2} \xi_{2}+\mathrm{B}_{3} \cos \lambda_{2} \xi_{1}+\mathrm{C}_{3} \sinh \lambda_{2} \xi_{2}+\mathrm{D}_{3} \cosh \lambda_{2} \xi_{2}-\xi_{3} \mathrm{v}_{1}(1)$

Where $\mathrm{Ai}, \mathrm{Bi}, \mathrm{Ci}$ and $\mathrm{Di}$ are arbitrary constants, which are determined from the boundary conditions.

The boundary conditions are at the fixed and free end, continuity of displacement moment, shear forces at the crack location, jump condition in the slope and compatibility conditions can be written as follows

The boundary conditions at the fixed end are as follows,

At $\quad \xi_{1}=0$ both displacement and slope are zero.

$$
\begin{aligned}
& \mathrm{V}_{1}(0)=0 \\
& \mathrm{~V}_{1}(0)=0
\end{aligned}
$$

The boundary conditions

$$
\begin{aligned}
& \text { At } \xi_{1}=1 \text { and } \xi_{1}=0 \quad \text { is given by } \\
& \mathrm{V}_{2}(0)=0 \\
& \mathrm{~V}_{2}(0)=0 \\
& \lambda_{1}^{4} \rho L_{0} \int^{1} \varepsilon_{2}\left\{V_{2}+\varepsilon_{2} \mathrm{~V}_{1}(1)\right\} d \varepsilon_{2}+\lambda_{1}^{4} M_{1} L^{2}\left\{V_{1}(1)+\left(V_{2}(1)\right\}-V_{1}^{\prime \prime}(1)=0\right. \\
& \lambda_{1}^{4} \rho L^{2} \int_{0}^{1}\left\{1 / L V_{2}(1)\right\} d \varepsilon_{2}+\lambda_{1}^{4} M_{1} L^{2}\left\{1 / L V_{2}(1)\right\}-V_{2}^{\prime \prime}(1)=0
\end{aligned}
$$

The compatibility conditions of the displacement, slope, moment and shear forces at the crack location are

$$
\text { At } \xi_{1}=\beta
$$

The continuity of the deflection at the crack is given by

$$
\mathrm{V}_{2}\left(\beta_{2}\right)-\mathrm{V}_{2}\left(\beta_{2}\right)=0
$$

At the crack location, the slope of deflection is discontinuous, the values of the slope at both sides of the crack are related to the crack stiffness by

$$
V_{2}\left(\beta_{2}\right)+\left(E_{2} I_{2} / K_{1} L_{2}\right) V_{2} "\left(\beta_{2}\right)-V_{3}{ }^{\prime}\left(\beta_{2}\right)=0
$$

From the theory of linear elastic fracture mechanics, the rotational spring stiffness $K_{t}$ is given by $K_{t}=$ $\mathrm{E}_{2} \mathrm{bh}^{2} / 72 \pi(\mathrm{a} / \mathrm{h})^{2} \mathrm{f}(\mathrm{a} / \mathrm{h})$

Where

$\mathrm{F}(\mathrm{a} / \mathrm{h})=0.6384-1.035(\mathrm{a} / \mathrm{h})+3.720 \mathrm{I}(\mathrm{a} / \mathrm{h})^{2}-5.1773(\mathrm{a} / \mathrm{h})^{3}+7.553(\mathrm{a} / \mathrm{h})^{5}+2.4909(\mathrm{a} / \mathrm{h})^{6}$

Where $\mathrm{K}=\left(\mathrm{K}_{\mathrm{t}} \mathrm{L}_{2} / \mathrm{E}_{2} \mathrm{I}_{2}\right)$ is the non-dimensional stiffness of the rotational spring representing the crack.

The internal moment at the crack location is same for the two segments and that leads to 


$$
\mathrm{V}_{2},\left(\beta_{2}\right)-\mathrm{V}_{3} "\left(\beta_{2}\right)=0
$$

The shear compatibility equation at the crack location is given by

$$
\mathrm{V}_{2}{ }^{\prime \prime}\left(\beta_{2}\right)-\mathrm{V}_{3}{ }^{\prime \prime}\left(\beta_{2}\right)=0
$$

The boundary conditions at

$$
\begin{aligned}
& \text { At } \varepsilon_{2}=1 \text { with end mass is given by } \\
& \mathrm{V}_{3}{ }^{\prime}(1)=0 \\
& \lambda_{2}{ }^{4} \mathrm{M}_{\mathrm{t}}\left\{\mathrm{V}_{3}(1)+\mathrm{V}_{1}(1)\right\}+\rho L \mathrm{~V}_{3}{ }^{\prime \prime}(1)=0
\end{aligned}
$$

Using the above boundary and compatibility conditions twelve homogeneous equations involving the unknown arbitrary constants are obtained. These can be expressed conveniently in the following form

$[A]_{12 \times 12}\{q\}_{12 \times 1}=\{0\}_{12 \times 1}$

Where

$$
\begin{aligned}
& \{\mathrm{q}\}=\left[\mathrm{A}_{1}, \mathrm{~B}_{1}, \mathrm{C}_{1}, \mathrm{D}_{1}, \mathrm{~A}_{2}, \mathrm{~B}_{2}, \mathrm{C}_{2}, \mathrm{D}_{2}, \mathrm{~A}_{3}, \mathrm{~B}_{3}, \mathrm{C}_{3}, \mathrm{D}_{3},\right]^{\mathrm{T}} \\
& \begin{array}{c}
\lambda_{1}^{4} \rho L \int_{0}^{1} \varepsilon 2\left\{V_{3}+\varepsilon_{2} V_{2}(1)\right\} d \varepsilon_{2}+\lambda_{1}^{4} M_{z} L^{2}\left\{V_{2}(1)+\left(V_{3}(1)\right\}-V_{2}^{\prime \prime}(1)=0\left(V_{3}(1)\right\}-V_{2}^{\prime \prime}(1)\right. \\
=0
\end{array} \\
& \lambda_{1}^{4} \rho L^{2} \int_{0}^{1}\left\{1 / L V_{2}(1)\right\} d \varepsilon_{2}+\lambda_{1}^{4} M_{z}\left\{1 / L V_{2}(1)\right\}+V_{2}{ }^{\prime \prime}(1)=0
\end{aligned}
$$

The boundary conditions at

At $=\varepsilon 2=1$ with end mass is given by

$\mathrm{V}_{3}(1)=0$

$$
\lambda_{1}^{4} M_{t}\left\{V_{3}(1)+V_{2}(1)\right\}+\rho L\left\{V_{3}(1)=0\right.
$$

Using the above boundary and compatibility conditions twelve homogeneous equations involving the unknown arbitrary constants are obtained. These can be expressed conveniently in the following form.

$[A]_{12 * 12}\{q\}_{12 * 1}=\{0\}_{12 * 1}$

Where,

$$
\{q\}=\left[A_{1}, B_{1}, C_{1}, D_{1}, A_{2}, B_{2}, C_{2}, D_{2}, A_{3}, B_{3}, C_{3} D_{3}\right]^{T}
$$

The non-zero elements of the matrix $[\mathrm{A}]$ are listed below. The terms contain the natural frequency parameters $\lambda$. For a non-trivial solution for $\{q\}$

$\operatorname{Det}[\mathrm{A}]=0$

This gives the characteristic equation. 


\section{RESULTS AND DISCUSSIONS}

The haracteristic equations are solved using MAT Lab 7.0.1 programming computational package to obtain the natural frequencies.

For numerical case study the below material properties and dimensions of the structure are taken up. The dimensions of the beam structure are length $\mathrm{L}_{1}=0.9 \mathrm{~m}, \mathrm{~L}_{2}=0.6 \mathrm{~m}$, width $\mathrm{b}=0.03 \mathrm{~m}$, depth $\mathrm{h}=0.05 \mathrm{~m}$. The material properties used for calculation material as aluminum with modulus of elasticity $E_{1}=E_{2}=70$ Gpa, density $\rho_{1}=\rho_{2}=260$ $\mathrm{kg} / \mathrm{m}^{3}$

The system ithout and with end mass and the angle between the beams is $90^{\circ}$. The natural frequencies are obtained by solving for the roots of the frequency equation are tabulated in tables 1 to 6 .

The frequency ratio is evaluated for different sets of crack parameters and a series of end mass rations. The frequency ratio in percentage is defined as

$\mathrm{r} \omega=\omega($ cracked $)-\omega($ uncracked $) / \omega($ uncracked $) X 100$

Table 1: Natural Frequencies of L-Frame with Crack on Segment Number1 (Vertical Segment Without End Mass)

\begin{tabular}{|c|c|c|c|c|c|c|c|c|c|}
\hline \multirow{2}{*}{$\begin{array}{c}\text { Crack } \\
\text { Location } \\
\left(\mathrm{L}_{\mathrm{c}} / \mathrm{L}_{\mathrm{i}}\right)\end{array}$} & \multirow{2}{*}{$\begin{array}{c}\text { Crack } \\
\text { Size } \\
(\mathrm{a} / \mathrm{h}) \\
\end{array}$} & \multicolumn{8}{|c|}{ Natural Frequencies (rad/sec) } \\
\hline & & $\omega_{1}$ & $\%$ Diff & $\omega_{2}$ & $\%$ Diff & $\omega_{3}$ & $\%$ Diff & $\omega_{4}$ & $\%$ Diff \\
\hline \multicolumn{2}{|c|}{ No Crack } & 149.730 & & 487.830 & & 1892.850 & & 3714.510 & \\
\hline 0.20 & 0.10 & 149.081 & -0.43 & 487.51 & -0.066 & 1892.623 & -0.012 & 3714.233 & -0.007 \\
\hline 0.20 & 0.20 & 147.279 & -1.634 & 486.625 & -0.247 & 1891.987 & -0.046 & 3713.467 & -0.028 \\
\hline 0.20 & 0.30 & 144.201 & -3.689 & 485.159 & -0.548 & 1890.927 & -0.102 & 3712.166 & -0.063 \\
\hline 0.20 & 0.40 & 139.561 & -6.783 & 483.058 & -0.979 & 1889.392 & -0.183 & 3710.225 & -0.115 \\
\hline 0.20 & 0.50 & 133.264 & -10.994 & 480.39 & -1.526 & 1887.414 & -0.287 & 3707.623 & -0.185 \\
\hline 0.20 & 0.60 & 125.598 & -16.114 & 477.42 & -2.134 & 1885.175 & -0.406 & 3704.529 & -0.269 \\
\hline 0.40 & 0.10 & 149.341 & -0.256 & 487.822 & -0.002 & 1888.945 & -0.206 & 3711.099 & -0.092 \\
\hline 0.40 & 0.20 & 149.256 & -0.981 & 487.795 & -0.008 & 1878.04 & -0.783 & 3701.632 & -0.347 \\
\hline 0.40 & 0.30 & 146.368 & -2.242 & 487.749 & -0.017 & 1859.522 & -1.761 & 3685.724 & -0.775 \\
\hline 0.40 & 0.40 & 143.439 & -4.199 & 487.678 & -0.031 & 1831.94 & -3.218 & 3662.448 & -1.402 \\
\hline 0.40 & 0.50 & 139.278 & -6.977 & 487.581 & -0.051 & 1795.073 & -5.166 & 3632.184 & -2.216 \\
\hline 0.40 & 0.60 & 133.938 & -10.544 & 487.46 & -0.076 & 1751.515 & -7.467 & 3597.764 & -3.143 \\
\hline 0.60 & 0.10 & 149.528 & -0.132 & 487.35 & -0.099 & 1887.381 & -0.289 & 3714.495 & -0.0001 \\
\hline 0.60 & 0.20 & 148.967 & -0.506 & 485.99 & -0.378 & 1872.192 & -1.091 & 3714.46 & -0.001 \\
\hline 0.60 & 0.30 & 147.975 & -1.169 & 483.626 & -0.862 & 1846.669 & -2.44 & 3714.402 & -0.003 \\
\hline 0.60 & 0.40 & 146.395 & -2.224 & 479.966 & -1.612 & 1809.253 & -4.417 & 3714.316 & -0.005 \\
\hline 0.60 & 0.50 & 144.064 & -3.781 & 474.794 & -2.673 & 1760.293 & -7.003 & 3714.203 & -0.008 \\
\hline 0.60 & 0.60 & 140.916 & -5.884 & 468.214 & -4.021 & 1703.871 & -9.984 & 3714.074 & -0.012 \\
\hline 0.80 & 0.10 & 149.645 & -0.053 & 468.448 & -0.284 & 1892.549 & -0.016 & 3709.491 & -0.135 \\
\hline 0.80 & 0.20 & 149.416 & -0.206 & 482.563 & -1.08 & 1891.697 & -0.061 & 3695.384 & -0.515 \\
\hline 0.80 & 0.30 & 149.005 & -0.481 & 475.878 & -2.45 & 1890.234 & -0.138 & 3671.091 & -1.169 \\
\hline 0.80 & 0.40 & 148.339 & -0.926 & 465.701 & -4.536 & 1888.017 & -0.255 & 3634.181 & -2.163 \\
\hline 0.80 & 0.50 & 147.324 & -1.603 & 451.645 & -7.418 & 1884.974 & -0.416 & 3583.672 & -3.522 \\
\hline 0.80 & 0.60 & 145.893 & -2.559 & 434.274 & -10.979 & 1881.25 & -0.613 & 3522.635 & -5.166 \\
\hline
\end{tabular}


Table 2: Natural Frequencies of L-Frame with Crack on Segment Number 1 (Vertical Segment) with End Mass $=0.4 \mathrm{Kg}$

\begin{tabular}{|c|c|c|c|c|c|c|c|c|c|}
\hline \multirow{2}{*}{$\begin{array}{c}\text { Crack } \\
\text { Locati } \\
\text { on } \\
\left(\mathrm{L}_{\mathrm{c}} / \mathrm{L}_{\mathrm{i}}\right)\end{array}$} & \multirow{2}{*}{$\begin{array}{c}\text { Crack } \\
\text { Size } \\
(\mathbf{a} / \mathbf{h})\end{array}$} & \multicolumn{8}{|c|}{ Natural Frequencies (rad/sec) } \\
\hline & & $\omega_{1}$ & $\%$ Diff & $\omega_{2}$ & $\%$ Diff & $\omega_{3}$ & $\%$ Diff & $\omega_{4}$ & $\%$ Diff \\
\hline \multicolumn{2}{|c|}{ No Crack } & 111.827 & & 318.9163 & & 1733.4130 & & 2943.8490 & \\
\hline 0.20 & 0.10 & 114.455 & -0.383 & 318.574 & -0.107 & 1733.089 & -0.019 & 2943.82 & -0.01 \\
\hline 0.20 & 0.20 & 110.253 & -1.457 & 317.63 & -0.403 & 1732.192 & -0.07 & 2943.738 & -0.04 \\
\hline 0.20 & 0.30 & 108.187 & -3.303 & 316.066 & -0.894 & 1730.698 & -0.157 & 2943.601 & -0.008 \\
\hline 0.20 & 0.40 & 105.046 & -6.11 & 313.822 & -1.597 & 1728.535 & -0.281 & 2943.4 & -0.015 \\
\hline 0.20 & 0.50 & 100.715 & -9.982 & 310.97 & -2.492 & 1725.752 & -0.442 & 2943.137 & -0.024 \\
\hline 0.20 & 0.60 & 95.365 & -14.763 & 307.794 & -3.487 & 1722.604 & -0.624 & 2942.833 & -0.035 \\
\hline 0.40 & 0.10 & 111.596 & -0.256 & 318.91 & -0.002 & 1730.481 & -0.169 & 2941.21 & -0.09 \\
\hline 0.40 & 0.20 & 110.787 & -0.979 & 318.894 & -0.007 & 1722.268 & -0.643 & 2933.907 & -0.338 \\
\hline 0.40 & 0.30 & 109.379 & -2.238 & 318.865 & -0.016 & 1708.227 & -1.453 & 2921.714 & -0.752 \\
\hline 0.40 & 0.40 & 107.194 & -4.191 & 318.821 & -0.03 & 1687.094 & -2.672 & 2904.036 & -1.352 \\
\hline 0.40 & 0.50 & 104.091 & -6.964 & 318.761 & -0.049 & 1658.444 & -4.325 & 2881.308 & -2.124 \\
\hline 0.40 & 0.60 & 100.108 & -10.524 & 318.688 & -0.072 & 1624.01 & -6.311 & 2855.764 & -2.992 \\
\hline 0.60 & 0.10 & 111.707 & -0.157 & 318.754 & -0.051 & 1728.274 & -0.297 & 2943.307 & -0.018 \\
\hline 0.60 & 0.20 & 111.207 & -0.604 & 318.296 & -0.195 & 1713.978 & -1.121 & 2941.821 & -0.069 \\
\hline 0.60 & 0.30 & 110.326 & -1.391 & 317.501 & -0.444 & 1689.865 & -2.512 & 2939.38 & -0.152 \\
\hline 0.60 & 0.40 & 108.932 & -2.637 & 316.272 & -0.829 & 1654.308 & -4.564 & 2935.924 & -0.269 \\
\hline 0.60 & 0.50 & 106.892 & -4.461 & 314.541 & -1.372 & 1607.401 & -7.27 & 2931.609 & -0.416 \\
\hline 0.60 & 0.60 & 104.17 & -6.893 & 312.346 & -2.06 & 1552.794 & -10.42 & 2926.906 & -0.576 \\
\hline 0.80 & 0.10 & 111.787 & -0.086 & 318.222 & -0.218 & 1732.557 & -0.049 & 2941.505 & -0.08 \\
\hline 0.80 & 0.20 & 111.511 & -0.332 & 316.272 & -0.829 & 1730.149 & -0.188 & 2934.934 & -0.303 \\
\hline 0.80 & 0.30 & 111.021 & -0.77 & 312.913 & -1.883 & 1725.006 & -0.427 & 2923.676 & -0.685 \\
\hline 0.80 & 0.40 & 110.229 & -1.478 & 307.79 & -3.489 & 1719.701 & -0.791 & 2906.684 & -1.262 \\
\hline 0.80 & 0.50 & 109.036 & -2.545 & 300.699 & -5.712 & 1711.003 & -1.293 & 2883.563 & -2.048 \\
\hline 0.80 & 0.60 & 107.378 & -4.026 & 291.921 & -8.465 & 1700.295 & -1.911 & 2855.68 & -2.995 \\
\hline
\end{tabular}

Table 3: Natural Frequencies of L-Frame with Crack on Segment Number 1 (Vertical Segment) with End Mass $=0.8 \mathrm{Kg}$

\begin{tabular}{|c|c|c|c|c|c|c|c|c|c|}
\hline \multirow{2}{*}{$\begin{array}{c}\text { Crack } \\
\text { Location } \\
\left(\mathrm{L}_{\mathrm{c}} / \mathrm{L}_{\mathrm{i}}\right)\end{array}$} & \multirow{2}{*}{$\begin{array}{c}\text { Crack } \\
\text { Size } \\
\text { (a/h) }\end{array}$} & \multicolumn{8}{|c|}{ Natural frequencies (rad/sec) } \\
\hline & & $\omega_{1}$ & $\%$ Diff & $\omega_{2}$ & $\begin{array}{c}\% \\
\text { Diff }\end{array}$ & $\omega_{3}$ & $\begin{array}{c}\% \\
\text { Diff }\end{array}$ & $\omega_{4}$ & $\%$ Diff \\
\hline \multicolumn{2}{|c|}{ No Crack } & 92.7908 & & 262.0426 & & 1689.0628 & & 2852.0465 & \\
\hline 0.20 & 0.10 & 91.514 & -1.38 & 260.798 & -0.47 & 1689.713 & -0.02 & 2851.982 & $\begin{array}{l}-0.00220 \\
-0.00506\end{array}$ \\
\hline 0.20 & 0.20 & 89.89 & -3.13 & 259.283 & -1.05 & 1686.061 & -0.08 & 2851.902 & 0.00506 \\
\hline 0.20 & 0.30 & 87.41 & -5.80 & 257.105 & -1.88 & 1683.67 & -0.18 & 2851.785 & -0.01 \\
\hline 0.20 & 0.40 & 83.968 & -9.51 & 254.329 & -2.94 & 1680.593 & -0.32 & 2851.633 & -0.01 \\
\hline 0.20 & 0.50 & 79.687 & -14.12 & 251.23 & -4.13 & 1677.113 & -0.50 & 2851.457 & -0.01 \\
\hline 0.20 & 0.60 & 92.556 & -0.25 & 262.028 & -0.01 & 1686.357 & -.0 .71 & 2849.453 & -0.02 \\
\hline 0.40 & 0.10 & 91.892 & -0.97 & 261.985 & -0.02 & 1678.773 & -0.16 & 2842.27 & -0.090 \\
\hline 0.40 & 0.20 & 90.736 & -2.21 & 261.913 & -0.05 & 1665.796 & -0.61 & 2830.269 & -0.34 \\
\hline 0.40 & 0.30 & 88.941 & -4.15 & 261.804 & -0.09 & 1646.23 & -1.38 & 2812.85 & -0.76 \\
\hline 0.40 & 0.40 & 86.391 & -6.90 & 261.654 & -0.15 & 1619.642 & -2.54 & 2790.417 & -1.37 \\
\hline 0.40 & 0.50 & 83.114 & -10.43 & 261.47 & -0.22 & 1587.586 & -4.11 & 2765.151 & -2.16 \\
\hline 0.40 & 0.60 & 92.637 & -0.197 & 261.945 & -0.04 & 1686.024 & -6.01 & 2851.396 & -3.05 \\
\hline 0.60 & 0.10 & 92.199 & -0.64 & 261.669 & -0.14 & 1670.003 & -0.30 & 2849.613 & -0.02 \\
\hline 0.60 & 0.20 & 91.428 & -1.47 & 261.19 & -0.33 & 1646.335 & -1.13 & 2846.69 & -0.09 \\
\hline 0.60 & 0.30 & 90.211 & -2.278 & 260.452 & -0.61 & 1611.396 & -2.53 & 2842.565 & -0.19 \\
\hline 0.60 & 0.40 & 88.436 & -4.69 & 259.412 & -1.00 & 1565.232 & -4.60 & 2837.434 & -0.33 \\
\hline 0.60 & 0.50 & 86.078 & -7.23 & 258.095 & -1.51 & 1511.385 & -7.33 & 2831.867 & -0.51 \\
\hline
\end{tabular}




\begin{tabular}{|l|l|l|l|l|l|l|l|l|l|}
\hline 0.60 & 0.60 & 92.698 & -0.10 & 261.532 & -0.19 & 1688.058 & $\begin{array}{c}- \\
10.52\end{array}$ & 2849.949 & -0.71 \\
\hline 0.80 & 0.10 & 92.433 & -0.39 & 260.096 & -0.74 & 1685.232 & -0.06 & 2844.072 & -0.07 \\
\hline 0.80 & 0.20 & 91.961 & -0.89 & 257.623 & -1.69 & 1680.37 & -0.23 & 2834.016 & $\begin{array}{c}-0.28 \\
28-0 .\end{array}$ \\
\hline 0.80 & 0.30 & 91.203 & -1.71 & 253.852 & -3.13 & 1672.969 & -0.51 & 2818.862 & -0.63 \\
\hline 0.80 & 0.40 & 90.066 & -2.94 & 248.634 & -5.12 & 1662.762 & -0.95 & 2798.29 & -1.16 \\
\hline 0.80 & 0.50 & 88.499 & -4.63 & 242.178 & -7.58 & 1650.197 & -1.56 & 2773.539 & -1.88 \\
\hline 0.80 & 0.60 & 88.499 & -4.63 & 242.178 & -7.58 & 1650.197 & -2.30 & 2773.539 & -2.75 \\
\hline
\end{tabular}

Table 4: Natural Frequencies of L-Frame with Crack on Segment Number 2 (Horizontal Segment) Without End Mass

\begin{tabular}{|c|c|c|c|c|c|c|c|c|c|}
\hline \multirow{2}{*}{$\begin{array}{c}\text { Crack } \\
\text { Location } \\
\left(L_{c} / L_{2}\right)\end{array}$} & \multirow{2}{*}{$\begin{array}{l}\text { Crack } \\
\text { Size } \\
(\mathrm{a} / \mathrm{h})\end{array}$} & \multicolumn{8}{|c|}{ Natural frequencies (rad/sec) } \\
\hline & & $\omega_{1}$ & $\%$ Diff & $\omega_{2}$ & \% Diff & $\omega_{3}$ & \% Diff & $\omega_{4}$ & $\%$ Diff \\
\hline \multicolumn{2}{|c|}{ No Crack } & 149.730 & & 487.830 & & 1892.850 & & 3714.510 & \\
\hline 0.20 & 0.10 & 149.725 & -0.0040 & 487.596 & -0.05 & 1880.419 & -0.66 & 3650.576 & -1.72 \\
\hline 0.20 & 0.20 & 149.724 & -0.0040 & 486.915 & $\begin{array}{l}-0.19 \\
\end{array}$ & 1843.363 & -2.61 & 3462.691 & -6.78 \\
\hline 0.20 & 0.30 & 149.723 & -0.0047 & 485.682 & -0.44 & 1773.119 & -6.33 & 3171.275 & -14.62 \\
\hline 0.20 & 0.40 & 149.722 & -0.0054 & 483.636 & -0.86 & 1656.4 & -12.49 & 2873.851 & -22.63 \\
\hline 0.20 & 0.50 & 149.719 & -0.0073 & 480.421 & -1.52 & 1495.099 & -21.01 & 2657.861 & -28.45 \\
\hline 0.20 & 0.60 & 149.715 & -0.100 & 475.675 & -2.49 & 1319.216 & -30.31 & 2530.081 & -31.89 \\
\hline 0.40 & 0.10 & 149.725 & -0.0040 & 487.738 & -0.02 & 1887.0 .24 & $\begin{array}{c}-0.31 \\
\end{array}$ & 3639.148 & -2.03 \\
\hline 0.40 & 0.20 & 149.725 & -0.0040 & 487.468 & -0.07 & 1869.965 & -1.21 & 3462.091 & -6.80 \\
\hline 0.40 & 0.30 & 149.724 & -0.0040 & 486.981 & -0.17 & 1838.237 & -2.89 & 3234.656 & -12.92 \\
\hline 0.40 & 0.40 & 149.724 & -0.0040 & 486.18 & -0.34 & 1787.336 & -5.73 & 2995.328 & -19.36 \\
\hline 0.40 & 0.50 & 149.723 & -0.0047 & 484.94 & -0.59 & 1700.343 & -10.17 & 2781.651 & -25.11 \\
\hline 0.40 & 0.60 & 149.721 & -0.0060 & 483.145 & -0.96 & 1589.834 & -16.17 & 2620.325 & -29.46 \\
\hline 0.60 & 0.10 & 149.725 & -0.0040 & 487.809 & $\begin{array}{l}-0.00 \\
\end{array}$ & 1891.158 & $\begin{array}{l}-0.09 \\
\end{array}$ & 3679.872 & $\begin{array}{l}-0.93 \\
\end{array}$ \\
\hline 0.60 & 0.20 & 149.725 & -0.0040 & 487.743 & -0.02 & 1886.252 & -0.35 & 3590.555 & -3.34 \\
\hline 0.60 & 0.30 & 149.725 & 0.0040 & 487.625 & -0.04 & 1877.28 & $\begin{array}{l}-0.82 \\
\end{array}$ & 3457.879 & -6.91 \\
\hline 0.60 & 0.40 & 149.725 & 0.0040 & 487.431 & $\begin{array}{l}-0.08 \\
\end{array}$ & 1862.205 & -1.62 & 3290.696 & -11.41 \\
\hline 0.60 & 0.50 & 149.724 & 0.0040 & 487.134 & -0.14 & 1838.14 & -2.89 & 3104.412 & -16.42 \\
\hline 0.60 & 0.60 & 149.724 & 0.0040 & 486.709 & -0.23 & 1802.186 & -4.79 & 2920.714 & -21.37 \\
\hline 0.80 & 0.10 & 149.725 & 0.0040 & 487.83 & -0.0010 & 1892.703 & $\begin{array}{l}-0.01 \\
\end{array}$ & 3710.345 & $\begin{array}{c}-0.11 \\
\end{array}$ \\
\hline 0.80 & 0.20 & 149.725 & 0.0040 & 487.825 & -0.0028 & 1832.272 & -0.03 & 3698.542 & -0.43 \\
\hline 0.80 & 0.30 & 149.725 & 0.0040 & 487.816 & $\begin{array}{l}-0.00 \\
\end{array}$ & 1891.497 & -0.07 & 3677.842 & $\begin{array}{l}-0.99 \\
\end{array}$ \\
\hline 0.80 & 0.40 & 149.725 & 0.0040 & 487.802 & $\begin{array}{l}-0.01 \\
\end{array}$ & 1890.226 & $\begin{array}{l}-0.14 \\
\end{array}$ & 3645.321 & -1.86 \\
\hline 0.80 & 0.50 & 149.725 & 0.0040 & 487.779 & -0.01 & 1888.261 & -0.24 & 3598.209 & -3.13 \\
\hline 0.80 & 0.60 & 149.725 & 0.0040 & 487.747 & $\begin{array}{l}-0.02 \\
\end{array}$ & 1885.425 & $\begin{array}{l}-0.39 \\
\end{array}$ & 3536.024 & -4.81 \\
\hline
\end{tabular}

Table 5: Natural Frequencies of L-Frame with Crack on Segment Number 2 (Horizontal Segment) Without End Mass $=0.4 \mathrm{~kg}$

\begin{tabular}{|c|c|c|c|c|c|c|c|c|c|}
\hline \multirow{2}{*}{$\begin{array}{l}\text { Crack } \\
\text { Location } \\
\left(\mathbf{L}_{c} / \mathbf{L}_{2}\right)\end{array}$} & \multirow{2}{*}{$\begin{array}{c}\text { Crack } \\
\text { Size } \\
(\mathbf{a} / \mathbf{h})\end{array}$} & \multicolumn{8}{|c|}{ Natural frequencies $(\mathrm{rad} / \mathrm{sec})$} \\
\hline & & $\omega_{1}$ & $\%$ Diff & $\omega_{2}$ & $\begin{array}{c}\% \\
\text { Diff }\end{array}$ & $\omega_{3}$ & $\%$ Diff & $\omega_{4}$ & $\begin{array}{c}\% \\
\text { Diff }\end{array}$ \\
\hline \multicolumn{2}{|c|}{ No Crack } & 111.8827 & & 318.9163 & & 1733.411 & & 2943.8627 & \\
\hline 0.20 & 0.10 & 111.882 & -0.0006 & 318.665 & -0.08 & 1700.356 & -1.191 & 2920.604 & -0.79 \\
\hline 0.20 & 0.20 & 111.879 & -0.0033 & 317.94 & -0.31 & 1602.769 & -7.54 & 2824.436 & -4.06 \\
\hline 0.20 & 0.30 & 111.874 & -0.0177 & 316.627 & -0.72 & 1437.838 & -17.05 & 2623.609 & -10.88 \\
\hline 0.20 & 0.40 & 111.866 & -0.0149 & 314.451 & -1.40 & 1232.622 & -28.89 & 2452.751 & -16.68 \\
\hline 0.20 & 0.50 & 111.854 & -0.0356 & 311.043 & -2.47 & 1035.193 & -40.28 & 2358.166 & -19.90 \\
\hline 0.20 & 0.60 & 111.836 & -0.0417 & 306.05 & -4.03 & 873.499 & -49.61 & 2308.641 & -21.58 \\
\hline 0.40 & 0.10 & 111.882 & -0.0006 & 318.788 & -0.04 & 1711.846 & -1.24 & 2865.814 & -2.65 \\
\hline 0.40 & 0.20 & 111.881 & -0.0015 & 318.417 & -0.04 & 1650.001 & -4.81 & 2705.492 & -8.10 \\
\hline 0.40 & 0.30 & 111.878 & -0.0042 & 317.75 & -0.37 & 1543.956 & -10.93 & 2543.982 & -13.58 \\
\hline 0.40 & 0.40 & 111.874 & -0.0077 & 316.652 & -0.71 & 1396.452 & -19.44 & 2420.963 & -17.76 \\
\hline
\end{tabular}




\begin{tabular}{|c|c|c|c|c|c|c|c|c|c|}
\hline 0.40 & 0.50 & 111.868 & -0.0131 & 314.949 & -1.24 & 1229.102 & -29.09 & 2342.229 & -20.44 \\
\hline 0.40 & 0.60 & 111.859 & -0.0002 & 312.48 & -2.02 & 1069.656 & -38.29 & 2295.873 & -22.01 \\
\hline 0.60 & 0.10 & 111.883 & -0.0002 & 318.867 & -0.02 & 1723.109 & -0.59 & 2888.524 & -1.88 \\
\hline 0.60 & 0.20 & 111.882 & -0.0006 & 318.724 & -0.06 & 1693.496 & -2.30 & 2767.262 & -6.00 \\
\hline 0.60 & 0.30 & 111.881 & -0.0015 & 318.467 & -0.14 & 1640.913 & -5.34 & 2627.407 & -10.75 \\
\hline 0.60 & 0.40 & 111.88 & -0.0024 & 318.047 & -0.27 & 1559.512 & -10.03 & 2498.128 & -15.14 \\
\hline 0.60 & 0.50 & 111.877 & -0.0050 & 317.4 & -0.48 & 1449.917 & -16.35 & 2397.164 & $-18-57$ \\
\hline 0.60 & 0.60 & 111.874 & -0.0077 & 316.471 & -0.77 & 1324.481 & -23.59 & 2328.251 & -20.91 \\
\hline 0.80 & 0.10 & 111.883 & -0.0002 & 318.906 & -0.00 & 1730.959 & -0.14 & 2927.019 & -0.57 \\
\hline 0.80 & 0.20 & 111.883 & -0.0002 & 318.877 & -0.01 & 1723.904 & -0.55 & 2882.855 & -2.07 \\
\hline 0.80 & 0.30 & 111.882 & -0.0006 & 318.825 & -0.03 & 1711.197 & -1.28 & 2815.679 & -4.35 \\
\hline 0.80 & 0.40 & 111.882 & -0.0006 & 318.739 & -0.06 & 1690.411 & -2.48 & 2729.385 & -7.29 \\
\hline 0.80 & 0.50 & 111.882 & -0.0006 & 318.608 & -0.10 & 1658.669 & -4.31 & 2632.507 & -10.58 \\
\hline 0.80 & 0.60 & 111.881 & -0.0015 & 318.421 & -0.16 & 1614.289 & -6.87 & 2537.552 & -13.80 \\
\hline
\end{tabular}

Table 6: Natural Frequencies of L-Frame with Crack on Segment Number 2 (Horizontal Segment) Without End Mass $=0.8 \mathrm{~kg}$

\begin{tabular}{|c|c|c|c|c|c|c|c|c|c|}
\hline \multirow{2}{*}{$\begin{array}{c}\text { Crack } \\
\text { Location } \\
\left(\mathrm{L}_{\mathrm{c}} / \mathbf{L}_{2}\right)\end{array}$} & \multirow{2}{*}{$\begin{array}{c}\text { Crack } \\
\text { Size } \\
(\mathrm{a} / \mathrm{h})\end{array}$} & \multicolumn{8}{|c|}{ Natural Frequencies (rad/sec) } \\
\hline & & $\omega_{1}$ & $\%$ Diff & $\omega_{2}$ & $\%$ Diff & $\omega_{3}$ & $\begin{array}{c}\% \\
\text { Diff }\end{array}$ & $\omega_{4}$ & $\begin{array}{c}\% \\
\text { Diff }\end{array}$ \\
\hline \multicolumn{2}{|c|}{ No Crack } & 92.7908 & & 262.0426 & & 1689.0609 & & 2852.0674 & \\
\hline 0.20 & 0.10 & 92.79 & -0.0008 & 261.802 & -0.09 & 1638.072 & -3.02 & 2829.036 & -0.81 \\
\hline 0.20 & 0.20 & 92.786 & -0.0051 & 261.108 & -0.36 & 1490.225 & -11.77 & 2702.154 & -5.26 \\
\hline 0.20 & 0.30 & 92.779 & -0.0127 & 259.851 & -0.84 & 1269.807 & -24.82 & 2472.189 & -13.32 \\
\hline 0.20 & 0.40 & 92.768 & -0.0245 & 257.769 & -1.63 & 1042.76 & -38.26 & 2337.847 & -18.03 \\
\hline 0.20 & 0.50 & 90.752 & -0.0418 & 254.508 & -2.88 & 853.316 & -49.48 & 2273.275 & -20.29 \\
\hline 0.20 & 0.60 & 92.727 & -0.0687 & 249.741 & -4.69 & 710.482 & -57.94 & 2240.645 & -21.44 \\
\hline 0.40 & 0.10 & 92.79 & 0.0008 & 261.914 & -0.05 & 1654.346 & -2.06 & 2746.335 & -3.71 \\
\hline 0.40 & 0.20 & 92.788 & -0.0030 & 261.546 & -0.19 & 1556.823 & -7.83 & 2558.989 & -10.28 \\
\hline 0.40 & 0.30 & 92.785 & -0.0062 & 260.881 & -0.44 & 1403.375 & -16.91 & 2406.823 & -15.61 \\
\hline 0.40 & 0.40 & 92.779 & -0.0127 & 259.786 & -0.86 & 1218.672 & -27.85 & 2312.008 & -18.94 \\
\hline 0.40 & 0.50 & 92.77 & -0.0224 & 258.088 & -1.51 & 1037.208 & -38.59 & 2258.682 & -20.80 \\
\hline 0.40 & 0.60 & 90.758 & -0.0435 & 255.627 & -2.45 & 881.957 & -47.78 & 2229.16 & -21.84 \\
\hline 0.60 & 0.10 & 92.791 & -0.0002 & 261.99 & -0.02 & 1671.431 & -1.04 & 2773.515 & -2.75 \\
\hline 0.60 & 0.20 & 92.79 & -0.0008 & 261.839 & -0.08 & 1621.433 & -4.00 & 2622.603 & -8.04 \\
\hline 0.60 & 0.30 & 92.788 & -0.0030 & 261.567 & -0.18 & 1536.524 & -9.03 & 2477.034 & -13.15 \\
\hline 0.60 & 0.40 & 92.786 & -0.0051 & 261.122 & -0.35 & 1416.702 & -16.12 & 2365.399 & -17.06 \\
\hline 0.60 & 0.50 & 92.782 & -0.0094 & 260.436 & -0.61 & 1274.681 & -24.53 & 2291.439 & -19.66 \\
\hline 0.60 & 0.60 & 92.777 & -0.0148 & 259.451 & -0.99 & 1131.215 & -33.03 & 2246.362 & -21.24 \\
\hline 0.80 & 0.10 & 92.791 & -0.0021 & 262.031 & -0.0044 & 1684.486 & -0.27 & 2825.807 & -0.92 \\
\hline 0.80 & 0.20 & 90.791 & -0.0021 & 261.997 & -0.02 & 1671.373 & -1.05 & 2761.051 & -3.19 \\
\hline 0.80 & 0.30 & 92.79 & -0.0008 & 261.936 & -0.04 & 1647.955 & -2.43 & 2671.937 & -6.32 \\
\hline 0.80 & 0.40 & 92.79 & -0.0008 & 261.837 & -0.08 & 1610.396 & -4.66 & 2570.711 & -9.86 \\
\hline 0.80 & 0.50 & 92.789 & -0.0019 & 261.685 & -0.14 & 1555.283 & -7.92 & 2471.712 & -13.34 \\
\hline 0.80 & 0.60 & 92.788 & -0.0030 & 161.468 & -0.22 & 1483.229 & -12.19 & 2387.738 & -16.28 \\
\hline
\end{tabular}




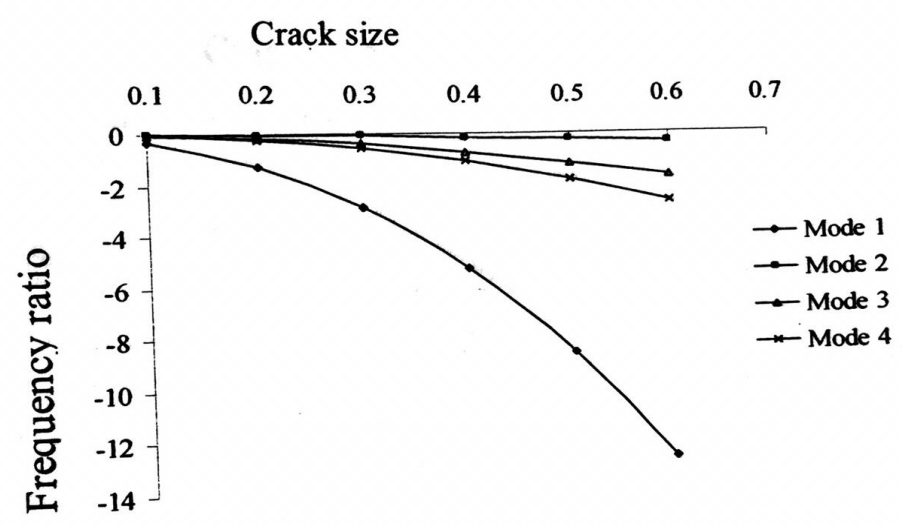

Figure 3: Plot of Frequency Ratio vs Crack Size for L-Frame with Crack in Segment Number One: $m=0, L_{c} / L_{l}=0.4$

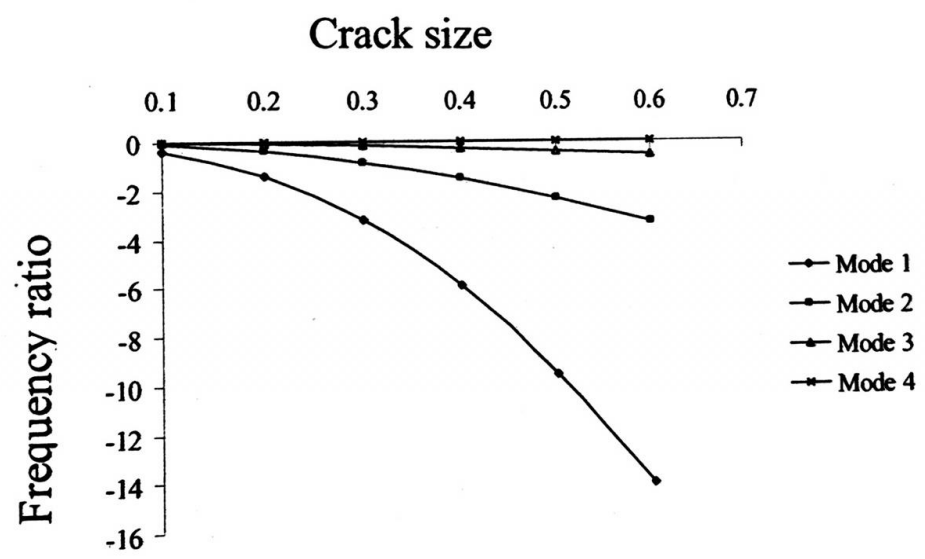

Figure 4: Plot of Frequency Ratio vs Crack Size for L-Frame with Crack in Segment Number One: $m=0.4, L_{c} / L_{I}=0.2$.

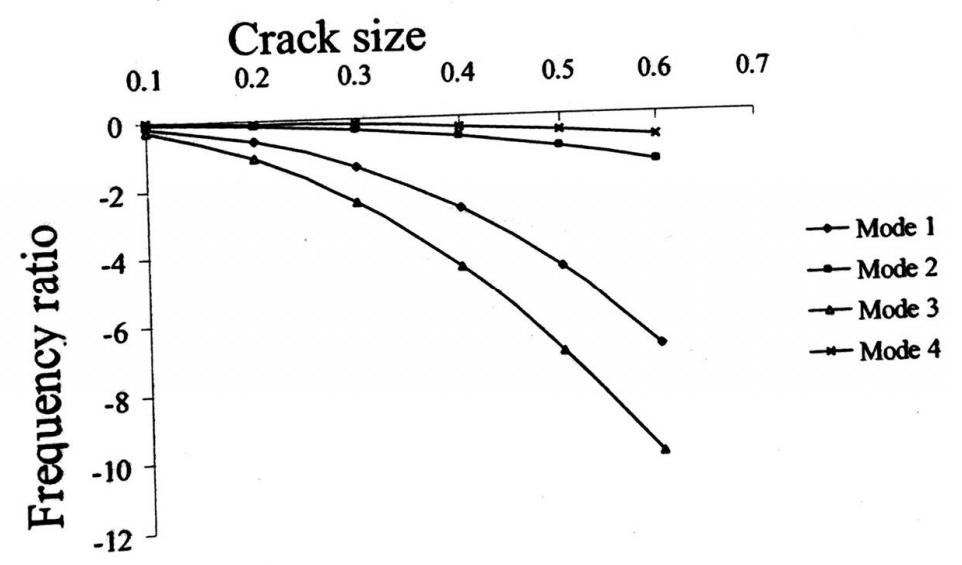

Figure 5: Plot of Frequency Ratio vs Crack Size for L-Frame with Crack in Segment Number One: $m=0.8, L_{c} / L_{I}=0.6$. 


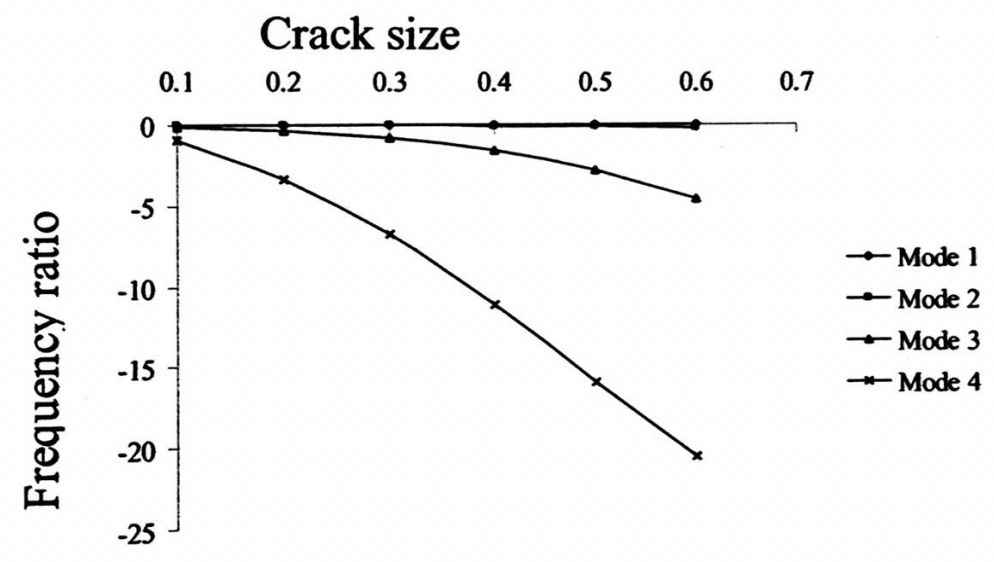

Figure 6: Plot of Frequency Ratio vs Crack Size for L-Frame with Crack in Segment Number One: $m=0, L_{c} / L_{2}=0.6$.

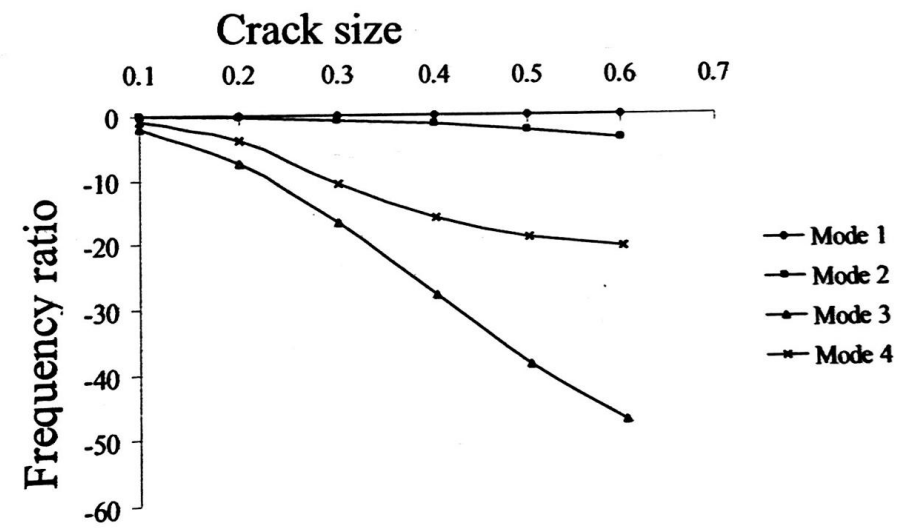

Figure 7: Plot of Frequency Ratio vs Crack Size for L-Frame with Crack in Segment Number One: $m=0.4, L_{c} / L_{2}=0.2$.

Table 1 presents the results of L-frame with crack on vertical segment without end mass. Here four different crack locations are considered i,e., $\mathrm{L}_{\mathrm{c}} / \mathrm{L}_{\mathrm{i}}=0.2,0.4,0.6$ and 0.8 . for each crack locations, crack sizes $(\mathrm{a} / \mathrm{h})$ varies from 0.1 to 0.6 . The natural frequency decreases with the increasing the crack size in all the four locations in mode 1 . In mode 2 also the natural frequency decreases with increasing crack size, but in location 0.4 there is no considerable decrease in the natural frequency. At crack location 0.6, in mode 4 there is no considerable decrease in the natural frequency with crack size. For crack location 0.8 the natural frequency decreases considerably and these are shown in Fig. 3

Table 2 and 3 presents the results of L-frame with crack on vertical segment with end mass $0.4 \mathrm{~kg}$ and $0.8 \mathrm{~kg}$ respectively with the increasing the end mass, the natural frequency decreases. As discussed earlier here also the natural frequency decreases in all the four modes, and these are shown in Fig $4 \& 5$.

Table 4 depicts the results of L-frame with crack on horizontal segment without end mass. Here also four different crack locations are considered. $\mathrm{I}, \mathrm{e} \mathrm{L}_{\mathrm{c}} / \mathrm{L}_{\mathrm{i}}=0.2,0.4,0.6$ and 0.8 . for each crack location the crack size $(\mathrm{a} / \mathrm{h}) \mathrm{varies}$ from 0.1 to 0.6. The natural frequency decreases with the increasing the crack size. But there is no considerable decrease in the natural frequency in mode 1,2, 3 and 4. Also the natural frequency decreases with increasing crack size. These are shown in Fig 6. 
Table 5 and 6 presents the results of L-frame with crack on horizontal segment with end mass $0.4 \mathrm{~kg}$ and $0.8 \mathrm{~kg}$ respectively. As discussed earlier, the natural frequency decreases with increasing end mass. There is no considerable decrease in natural frequency in mode 1, but in mode 2, 3 and 4 the natural frequency decreases considerably with increasing the crack size when compared to mode 1. These are shown in Fig. 7

By examining the above results, the following observations could be made.

- As expected, the natural frequency decreases with the introduce the crack in the frame.

- The frequency ratio decreases by increasing the crack size

- When increasing the end mass the natural frequency decreases.

- The frequency ratio is more sensitive to the crack size $(\mathrm{a} / \mathrm{h})$ than to the crack location $\left(\mathrm{L}_{\mathrm{c}} / \mathrm{L}_{\mathrm{i}}\right)$

\section{CONCLUSIONS}

The theoretical basis for a vibration based NDE technique for an assessment for a frame structure has been presented.

- The two member open frame structure was modeled using Euler-Bernoulli beam theory.

- The linearized equation of motion is derived using Hamilton's principle with crack condition.

- The transverse normal edge crack was modeled using the concept of rotational spring.

- The natural frequencies are determined by finding the first four roots of the characteristic equations.

- The numerical values of the natural frequencies are tabulated.

- The frequency ratio is more sensitive to the crack size than to the crack location.

- As expected the natural frequency decreases with the introduce of the crack in a structure.

\section{REFERENCES}

1. Chondros T G and Dimarogonas A D and J. Yao "A Continuous cracked beam vibration theory" Journal of sound and Vibration, 1998, 215 (1) pp 17-34.

2. Rizos $P F$ and Aspragathos $N$ and Dimarogonas A D, "identification of crack location and magnitude in a cantilever beam from the vibration modes", Journal of Sound and Vibration 1990 138(3), 381-388.

3. Dado M H "A Comprehensive crack identification algorithms for beams under different end conditions", Applied Acoustics, 51(1997) 381-398.

4. Jialou Hu Robert Y Liang, "An Integrated Approach to Detection of cracks using vibration characteristics" Journal of the Franklin institute Pergamom Press 1993, Vol 330. No. 56, pp 841-853

5. Nandwana B P and Maiti S K "Detection of the location and size of a crack in stepped cantilever beams-based on measurements of natural frequencies" Journal of Sound and Vibrations 1997

6. Nandwana B P and Maiti S K "Modelling of Vibration of beam in presence of inclined edge or internal crack for its possible detection based on frequency measurements" Engineering Fracture Mechanics 1997, 58(3), pp 193-205.

7. Chinchalkar S "Determination of Crack location in beams using natural frequencies", Journal of Sound and Vibration 2001, 247(3) pp $417-429$. 
8. Dado MH F, Abuzeid $O$ "coupled transverse and axial vibratory behaviour of cracked beam with end mass and rotary inertia", Journal of Sound and Vibration 2003, 261, pp 675-696.

9. Oguamanam D C D and Hansen $J S$ and Heppler $G R$, Vibration of orbitrily oriented two member frame with tip mass. Journal of sound and Vibration (1998)209(4) 651-669.

10. Robert Y Liang and Fred K Choy and Jialou Hu, "Detection of Cracks in Beam structures using measurements of natural frequencies” Journal of the Franklin Institute” Pergamom Press 1991, Vol 328 No. 4, pp 505-518

11. Chondros T G and Dimarogonas A D "Vibration of a cracked cantilever beam" Journal of Vibration and Acoustics July 1998, vol. 120, pp 742-746.

12. Lin HP, Ro J, "Vibration Analysis of Planar Serial-frame structures", Journal of sound and Vibration, 2003, 262, pp 11131131.

13. Rao S S, “Mechanical Vibrations” fourth Edition, 2004

14. Stanley T Rolfe and John M Barsom, "Fracture and Fatigue control in structures, Applications of Fracture Mechanics", 1997 by Prentice Hall, Inc, Englewood Cliffs, New Jersy, 07632

15. Muhsin J. Jeewg, Abdal-Kareem F. Hassan \& Jawad. K. Zeboon, "Experimental and Numerical Investigation of the Dynamic Characteristic of Laminated Composite Plate Hybrid with Steel “, International Journal of General Engineering and Technology (IJGET), Vol. 3, Issue 1, pp. 27-36

16. Mashitah Abu Bakar \& Jamali Md Sah, "Dynamic Response Analysis for Development of Flexible Lightweight Vehicle Chassis using CAE Tools “, International Journal of Mechanical Engineering (IJME), Vol. 7, Issue 3,pp. 9-20

17. Afrim Loku \& Fatlum Gogiqi, "Factors of Individual Behavior in an Organization and its Performance at Work", IMPACT: International Journal of Research in Business Management (IMPACT: IJRBM), Vol. 4, Issue 5, pp. 67-72

18. Shital Naikwade \& Nitin Sippy, "A Study of Consumer Behavior Pattern for Select Eye-Care Facilities in Vashi and Koparkhairne, Navi Mumbai “, BEST: International Journal of Humanities, Arts, Medicine and Sciences (BEST: IJHAMS), Vol. 3, Issue 9, pp. 11-24 

